\title{
Using the Distribution Theory to Simultaneously Calibrate the Sensors of a Mobile Robot
}

\author{
Agostino Martinelli \\ INRIA Rhone Alpes, Grenoble, FRANCE \\ email: agostino.martinelli@ieee.org
}

\begin{abstract}
This paper introduces a simple and very efficient strategy to extrinsically calibrate a bearing sensor (e.g. a camera) mounted on a mobile robot and simultaneously estimate the parameters describing the systematic error of the robot odometry system. The paper provides two contributions. The first one is the analytical computation to derive the part of the system which is observable when the robot accomplishes circular trejectories. This computation consists in performing a local decomposition of the system, based on the theory of distributions. In this respect, this paper represents the first application of the distribution theory in the frame-work of mobile robotics. Then, starting from this decomposition, a method to efficiently estimate the parameters describing both the extrinsic bearing sensor calibration and the odometry calibration is derived (second contribution). Simulations and experiments with the robot e-Puck equipped with encoder sensors and a camera validate the approach.
\end{abstract}

\section{INTRODUCTION}

A sensor calibration technique is a method able to estimate the parameters characterizing the systematic error of the sensor. In the last decades, this problem has been considered with special emphasis in the field of computer vision. The problem of camera calibration consists in estimating the intrinsic and extrinsic parameters based on a number of points whose object coordinates in a global frame are known and whose image coordinates are measured [19]. In robotics, when a camera is adopted, the calibration only regards the estimation of the extrinsic parameters, i.e. the parameters describing the configuration of the camera in the robot frame. In the case of robot wrists, very successful approaches are based on the solution of a homogeneous transform equation of the form $A X=X B$ which is obtained by moving the robot wrist and observing the resulting motion of the camera [15], [18]. In particular, in the previous equation, $A$ describes the configuration change of the wrist, $B$ the configuration change of the camera and $X$ the unknown camera configuration in the wrist reference frame. $A$ and $B$ are assumed to be known with high accuracy: $A$ is obtained by using the encoder data and $B$ by using the camera observations of a known object before and after the wrist movement [18]. In mobile robotics the situation changes dramatically and the previous methods cannot be applied. Unfortunately, the displacement of a mobile robot obtained by using the encoder data is not precise as in the case of a wrist. In other words, the previous matrix $A$ is roughly estimated by the encoders. A possible solution to this inconvenient could be obtained by adopting other sensors to estimate the robot movement (i.e. the matrix $A$ ).
However, most of times the objective is to estimate the camera configuration in the reference frame attached on the robot odometry system. Therefore, it is important to introduce a new method able to perform simultaneously the extrinsic camera calibration and the calibration of the robot odometry system. So far, the two problems have been considered separately.

\section{A. Previous Works}

Regarding the odometry, a very successful strategy has been introduced in 1996 by Borenstein and Feng [3]. This is the UMBmark method. It is based on absolute robot position measurements after the execution of several square trajectories. In [10] the problem of also estimating the non-systematic odometry errors was considered. More recently, a method based on two successive least-squares estimations has been introduced [2]. Finally, very few approaches calibrate the odometry without the need of an a priori knowledge of the environment and/or of the use of global position sensors (like a GPS) [6], [17], [20].

Regarding the problem of sensor to sensor calibration, several cases have recently been considered (e.g. IMU-camera [16], laser scanner-camera [4], [21], [22] and odometry-camera [12]). In [12], an observability analysis taking into account the system nonlinearities was also provided to understand whether the system contains the necessary information to perform the self calibration. Indeed, a necessary condition to perform the estimation of a state, is that the state is observable. In [12] it was investigated whether the state containing the extrinsic parameters of the vision sensor is or is not observable. The $o b$ servability rank criterion introduced by Hermann and Krener [8] was adopted to this scope. The same observability analysis was later applied to the case of the odometry self-calibration [13]. However, in these works, what it was determined is only whether the state containing the parameters defining the sensor systematic error is observable or not. On the other hand, when a state is not observable, suitable functions of its components could be observable and therefore could be estimated. The derivation of these functions is very important in order to properly exploit the information contained in the sensor data to estimate a given set of parameters. This derivation requires to perform a local decomposition [9]. While in the linear case this decomposition is easy to be performed, in the non linear case it is often troublesome and requires to apply the theory of distributions developed in [9]. 


\section{B. Paper Contributions}

In this paper we consider simultaneously the problems of odometry calibration and the extrinsic calibration of a bearing sensor. Furthermore, the calibration is carried out by only using a single point feature. To the best of our knowledge this problem has never been investigated before. The paper provides two contributions:

- A local decomposition of the considered system based on the theory of distributions developed in [9];

- A new strategy to robustly, efficiently and accurately calibrate both the bearing sensor and the odometry system.

The first contribution was preliminary discussed in [14] where the local decomposition has been performed only for the special cases of straight trajectory and pure rotation. To the best of our knowledge this contribution represents the first application of the distribution theory in the field of mobile robotics and the first non-trivial application of this theory to face a real estimation problem. In the specific case, it allows us to detect for circular trajectories which functions of the original calibration parameters are observable. Then, by performing at least three independent circular trajectories, it is possible to evaluate all the parameters describing our calibration problem.

Section II defines the calibration problem and provides the basic equations to characterize the system dynamics and the observation. In section III we remind some results from the theory developed in [8] (III-A) and [9] (III-B). In section IV we perform the decomposition for circular trajectories. This allows us to detect the functions of the parameters which are observable. Then, in section $\mathrm{V}$, we summarize some important analytical properties for the observation function whose derivation is available in [11]. Based on these properties, we introduce the calibration strategy in section VI whose validation is provided in VII through simulations and experiments. Conclusions are provided in section VIII.

\section{The CONSIDERED System}

We consider a mobile robot moving in a $2 D$-environment. The configuration of the robot in a global reference frame can be characterized through the vector $\left[x_{R}, y_{R}, \theta_{R}\right]^{T}$ where $x_{R}$ and $y_{R}$ are the cartesian robot coordinates and $\theta_{R}$ is the robot orientation. The dynamics of this vector are described by the following non-linear differential equations:

$$
\left[\begin{array}{l}
\dot{x}_{R}=v \cos \theta_{R} \\
\dot{y}_{R}=v \sin \theta_{R} \\
\dot{\theta}_{R}=\omega
\end{array}\right.
$$

where $v$ and $\omega$ are the linear and the rotational robot speed, respectively. The link between these velocities and the robot controls $(u)$ depends on the considered robot drive system. We will consider the case of a differential drive. In order to characterize the systematic odometry error we adopt the model introduced in [3]. We have:

$$
v=\frac{\delta_{R} v_{R}+\delta_{L} v_{L}}{2} \quad \omega=\frac{\delta_{R} v_{R}-\delta_{L} v_{L}}{\delta_{B} B}
$$

where $v_{R}$ and $v_{L}$ are the control velocities (i.e. $u=\left[v_{R}, v_{L}\right]^{T}$ ) for the right and the left wheel, $B$ is the nominal value for the distance between the robot wheels and $\delta_{R}, \delta_{L}$ and $\delta_{B}$ characterize the systematic odometry error due to an uncertainty on the wheels diameters and on the distance between the wheels.

Furthermore, a bearing sensor (e.g. a camera) is mounted on the robot. We assume that its vertical axis is aligned with the $z$-axis of the robot reference frame and therefore the transformation between the frame attached to this sensor and the one of the robot is characterized through the three parameters $\phi, \rho$ and $\psi$ (see fig. 1).

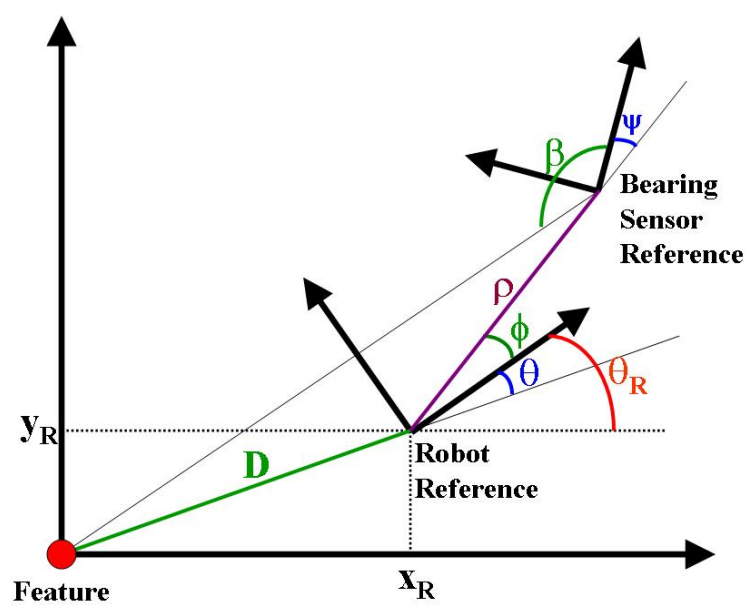

Fig. 1. The two frames attached to the robot and to the bearing sensor.

The available data are the control $u=\left[v_{R}, v_{L}\right]^{T}$ and the bearing angle of a single feature ( $\beta$ in fig. 1) at several time steps during the robot motion.

We introduce the following quantities:

$$
\mu \equiv \frac{\rho}{D} \equiv \frac{\rho}{\sqrt{x_{R}^{2}+y_{R}^{2}} ;} \quad \begin{aligned}
& \theta \equiv \theta_{R}-\operatorname{atan} 2\left(y_{R}, x_{R}\right) \\
& \gamma \equiv \theta+\phi
\end{aligned}
$$

By using simple trigonometry algebra we obtain (fig. 1):

$$
\beta= \begin{cases}-\operatorname{atan}\left(\frac{\sin \gamma}{\mu+\cos \gamma}\right)-\psi+\pi & \gamma_{-} \leq \gamma \leq \gamma_{+} \\ -\operatorname{atan}\left(\frac{\sin \gamma}{\mu+\cos \gamma}\right)-\psi & \text { otherwise }\end{cases}
$$

where $\gamma_{-}$and $\gamma_{+}$are the two solutions (in $[-\pi, \pi)$ ) of the equation $\cos \gamma=-\mu$ with $\gamma_{+}=-\gamma_{-}$and $\gamma_{+}>0$. We made the assumption $0<\mu<1$ since we want to avoid collisions between the robot and the feature $(D>\rho)$.

By using (1) and the definitions in (3) the dynamics of our system are described by the following equations: 


\begin{tabular}{|c|}
\hline Original Calibration Parameters \\
Camera: $\phi, \rho, \psi \quad$ Odometry: $\delta_{R}, \delta_{L}, \delta_{B}$ \\
\hline Observable Parameters \\
$\phi, \quad \psi, \quad \eta \equiv \frac{\delta_{R}}{2 \rho}, \quad \delta \equiv \frac{\delta_{L}}{\delta_{R}}, \quad \xi \equiv \frac{1}{B} \frac{\delta_{R}}{\delta_{B}}$ \\
\hline Parameters observable in a single q-trajectory \\
$A^{q} \equiv \frac{\Psi_{1}^{q}-\Psi_{3}}{1+\Psi_{1}^{q} \Psi_{3}}, V^{q} \equiv \Psi_{2}^{q} \frac{1+\Psi_{1}^{q} \Psi_{3}}{1+\Psi_{3}^{2}}, L^{q} \equiv \psi-\operatorname{atan} \Psi_{1}^{q}, \xi_{q} \equiv \xi(1-q \delta)$ \\
where: $\Psi_{1}^{q} \equiv \frac{\xi_{q}-\eta_{q} \sin \phi}{\eta_{q} \cos \phi}, \quad \Psi_{2}^{q} \equiv \frac{\mu \eta_{q} \cos \phi}{\sin \gamma}$, \\
$\Psi_{3} \equiv \frac{\mu+\cos \gamma}{\sin \gamma}, \quad \eta_{q} \equiv \eta(1+q \delta)$ \\
\hline
\end{tabular}

TABLE I

VARIABLES ADOPTED IN THIS PAPER

$$
\left\{\begin{array}{l}
\dot{\mu}=-\mu^{2} \frac{v}{\rho} \cos (\gamma-\phi) \\
\dot{\gamma}=\omega-\mu \frac{v}{\rho} \sin (\gamma-\phi) \\
\dot{\phi}=\dot{\rho}=\dot{\psi}=\dot{\delta}_{R}=\dot{\delta}_{L}=\dot{\delta}_{B}=0
\end{array}\right.
$$

The goal is to simultaneously estimate the parameters $\phi, \rho$, $\psi, \delta_{R}, \delta_{L}$ and $\delta_{B}$ using the available data (i.e. $v_{R}, v_{L}$ and $\beta$ in a given time interval). Since these data consists of angle measurements (the wheel diameters are not known and in fact are to be estimated), the best we can hope is the possibility to estimate these parameters up to a scale factor. In particular, we will refer to the following parameters:

$$
\phi, \quad \psi, \quad \eta \equiv \frac{\delta_{R}}{2 \rho}, \quad \delta \equiv \frac{\delta_{L}}{\delta_{R}}, \quad \xi \equiv \frac{1}{B} \frac{\delta_{R}}{\delta_{B}}
$$

whose dynamics are:

$$
\left\{\begin{array}{l}
\dot{\mu}=-\mu^{2} \eta\left(v_{R}+\delta v_{L}\right) \cos (\gamma-\phi) \\
\dot{\gamma}=\xi\left(v_{R}-\delta v_{L}\right)-\mu \eta\left(v_{R}+\delta v_{L}\right) \sin (\gamma-\phi) \\
\dot{\phi}=\dot{\psi}=\dot{\eta}=\dot{\delta}=\dot{\xi}=0
\end{array}\right.
$$

In section IV we derive for circular trajectories, which functions of $\mu, \gamma, \phi, \psi, \eta, \delta$ and $\xi$ are observable and hence can be estimated. Then, in section VI we introduce a very efficient strategy to estimate these parameters by considering three independent circular trajectories. Finally, by adding a simple metric measurement (e.g. the initial distance between the robot and the feature) the original parameters $\phi, \rho, \psi, \delta_{R}$, $\delta_{L}$ and $\delta_{B}$ can also be estimated.

For the sake of clarity we report all the variables appearing in this paper in table I.

\section{OBSERVABILITY PROPERTIES AND LOCAL DECOMPOSITION}

A general characterization for systems in the frame work of autonomous navigation is provided by the following equations:

$$
\left\{\begin{array}{l}
\dot{S}=\sum_{i=1}^{M} f_{i}(S) u_{i} \\
y=h(S)
\end{array}\right.
$$

where $S \in \Sigma \subseteq \Re^{n}$ is the state, $u_{i}$ are the system inputs, $y \in \Re$ is the output (we are considering a scalar output for the sake of clarity, the extension to a multi dimensions output is straightforward). The system defined by (4) and (7) can be characterized by (8). We have: $S=[\mu, \gamma, \phi, \psi, \eta, \delta, \xi]^{T}$, $M=2, u_{1}=v_{R}, u_{2}=v_{L}, f_{1}(S)=\left[-\mu^{2} \eta \cos (\gamma-\phi), \xi-\right.$ $\mu \eta \sin (\gamma-\phi), 0,0,0,0,0]^{T}, f_{2}(S)=-\left[\mu^{2} \eta \delta \cos (\gamma-\phi), \xi \delta+\right.$ $\mu \eta \delta \sin (\gamma-\phi), 0,0,0,0,0]^{T}$ and $y=\beta$.

\section{A. Observability Rank Criterion}

We want to remind some concepts in the theory by Hermann and Krener in [8]. We will adopt the following notation. We indicate the $k^{\text {th }}$ order Lie derivative of a scalar field $\Lambda$ along the vector fields $v_{i_{1}}, v_{i_{2}}, \ldots, v_{i_{k}}$ with $L_{v_{i_{1}}, v_{i_{2}}, \ldots, v_{i_{K}}}^{k} \Lambda$. We remind the definition of the Lie derivative. It is provided by the following two equations:

$$
\begin{gathered}
L^{0} \Lambda=\Lambda \\
L_{v_{i_{1}}, \ldots, v_{i_{k}}, v_{i_{k+1}}}^{k+1} \Lambda=\nabla_{S}\left(L_{v_{i_{1}}, \ldots, v_{i_{k}}}^{k} \Lambda\right) \cdot v_{i_{k+1}}
\end{gathered}
$$

where the symbol "." denotes the scalar product. Now, let us refer to the system in (8) and let us indicate with $\Omega$ the space of all the Lie derivatives $L_{f_{i_{1}}, \ldots, f_{i_{k}}}^{k} h,\left(i_{1}, \ldots, i_{k}=\right.$ $1, \ldots, M)$ where the functions $f_{i_{j}}(j=(1, \ldots, M))$ are the ones appearing in (8). Furthermore, we denote with $d L_{f_{i_{1}}, \ldots, f_{i_{k}}}^{k} h$ the gradient of the corresponding Lie derivative (i.e. $\left.d L_{f_{i_{1}}, \ldots, f_{i_{k}}}^{k} h \equiv \nabla_{S} L_{f_{i_{1}}, \ldots, f_{i_{k}}}^{k} h\right)$ and we denote with $d \Omega$ the space spanned by all these gradients.

In this notation, the observability rank criterion can be expressed in the following way: The dimension of the largest observable sub-system at a given $S_{0}$ is equal to the dimension of $d \Omega$. Therefore, the problem of understanding wheter a system is observable or not can be solved by computing the rank of the matrix whose lines are the gradients of the Lie derivatives.

\section{B. Local Decomposition}

Let us suppose that the system in (8) is not observable and that the dimension of the largest observable subsystem is $n_{o b s}$. According with the theory of distributions developed in [9], we can find $n_{o b s}$ independent functions of the components of the original state $S$ which are observable and $n-n_{o b s}$ independent functions of the components of $S$ which are not observable. More precisely, if we include the $n_{\text {obs }}$ observable functions in the vector $S_{b}$ and the other $n-n_{o b s}$ functions in the vector $S_{a}$, we have the following decomposition for the original system:

$$
\left\{\begin{array}{l}
\dot{S}_{a}=\sum_{i=1}^{M} f_{i}^{a}\left(S_{a}, S_{b}\right) u_{i} \\
\dot{S}_{b}=\sum_{i=1}^{M} f_{i}^{b}\left(S_{b}\right) u_{i} \\
y=h_{b}\left(S_{b}\right)
\end{array}\right.
$$


In particular, the subsystem defined by the last two equations in (10) is independent of the value of $S_{a}$ and it is observable. Therefore, by performing this decomposition, we can use the information coming from the dynamics (i.e. the knowledge of $u(t)$ ) and the observations $(y(t))$ in order to estimate the observable quantities $\left(S_{b}\right)$. This decomposition is very important in every estimation problem when the state is not observable. Indeed, estimating directly the original state $S$ results in an erroneous evaluation.

In section II, when we introduced $\mu$ and $\gamma$ (defined in (3)) and the three parameters $\eta, \delta, \xi$, we performed such a decomposition for the state $\left[x_{R}, y_{R}, \theta_{R}, \phi, \rho, \psi, \delta_{R}, \delta_{L}, \delta_{B}\right]^{T}$ : indeed, the new state $[\mu, \gamma, \phi, \psi, \eta, \delta, \xi]^{T}$ is observable as proven in [11] and its components are non linear functions of the components of the original state (which is not observable). On the other hand, in most of cases it is very troublesome to perform such a decomposition. In the next section we perform such a decomposition for the same state (i.e. $[\mu, \gamma, \phi, \psi, \eta, \delta, \xi]^{T}$ ) but when we only allow the robot to move along circular trajectories. We apply the distributions theory developed in [9].

\section{Local Decomposition for Circular TRAJECTORIES}

We consider the one-degree of freedom motion obtained by setting $v_{R}=\nu, v_{L}=q \nu$. Let us define:

$$
\eta_{q} \equiv \eta(1+q \delta) \quad \xi_{q} \equiv \xi(1-q \delta)
$$

From the dynamics in (7) we obtain the following dynamics:

$$
\left\{\begin{array}{l}
\dot{\mu}=-\mu^{2} \eta_{q} \nu \cos (\gamma-\phi) \\
\dot{\gamma}=\xi_{q} \nu-\mu \eta_{q} \nu \sin (\gamma-\phi) \\
\dot{\eta}_{q}=\dot{\xi}_{q}=\dot{\phi}=\dot{\psi}=0
\end{array}\right.
$$

In the next we provide the steps necessary to perform the decomposition of the system whose dynamics are given in (12) and whose output is the observation in (4). We proceed in two separate steps. We first consider the following simpler system:

$$
\left\{\begin{array}{l}
\dot{\mu}=-\mu^{2} \eta_{q} \nu \cos (\gamma-\phi) \\
\dot{\gamma}=\xi_{q} \nu-\mu \eta_{q} \nu \sin (\gamma-\phi) \\
\dot{\eta}_{q}=\dot{\xi}_{q}=\dot{\phi}=0 \\
y=\frac{\sin \gamma}{\mu+\cos \gamma}
\end{array}\right.
$$

where we removed the variable $\psi$. We apply the method developed in [9] in order to find the local decomposition for this simplified system.

The associated partial differential equation is in this case:

$\frac{\mu \cos \gamma+1}{\sin \gamma} \frac{\partial \Psi}{\partial \mu}+\frac{\partial \Psi}{\partial \gamma}+\frac{\xi_{q} \cos \phi}{\eta_{q} \mu \sin \gamma} \frac{\partial \Psi}{\partial \phi}+\frac{\xi_{q} \sin \phi-\eta_{q}}{\mu \sin \gamma} \frac{\partial \Psi}{\partial \eta_{q}}=0$

namely, every solution $\Psi\left(\mu, \gamma, \phi, \eta_{q}, \xi_{q}\right)$ of the previous partial differential equation is an observable quantity for the system in (13). We found the following four independent solutions:

$$
\begin{gathered}
\Psi_{1}^{q} \equiv \frac{\xi_{q}-\eta_{q} \sin \phi}{\eta_{q} \cos \phi}, \quad \Psi_{2}^{q} \equiv \frac{\mu \eta_{q} \cos \phi}{\sin \gamma}, \\
\Psi_{3} \equiv \frac{\mu+\cos \gamma}{\sin \gamma}, \quad \xi_{q}
\end{gathered}
$$

The local decomposition of (13) is:

$$
\left\{\begin{aligned}
\dot{\Psi}_{1}^{q} & =0 \\
\dot{\Psi}_{2}^{q} & =\nu \Psi_{2}^{q}\left(\Psi_{1}^{q} \Psi_{2}^{q}-\xi_{q} \Psi_{3}\right) \\
\dot{\Psi}_{3} & =\nu\left(\Psi_{2}^{q}+\Psi_{1}^{q} \Psi_{2}^{q} \Psi_{3}-\xi_{q}-\xi_{q} \Psi_{3}^{2}\right) \\
\dot{\xi}_{q} & =0 \\
y & =\frac{1}{\Psi_{3}}
\end{aligned}\right.
$$

Let us proceed with the second step. We add to the system in (15) the parameter $\psi$ (with $\dot{\psi}=0$ ) and we consider the output $y=\beta$ instead of $y=\frac{1}{\Psi_{3}}$. We apply again the method in [9] on the resulting system. The associated partial differential equation is in this case:

$\left(\Psi_{1}^{q^{2}}+1\right) \frac{\partial G}{\partial \Psi_{1}^{q}}+\left(\Psi_{2}^{q}\left(\Psi_{3}-\Psi_{1}^{q}\right)\right) \frac{\partial G}{\partial \Psi_{2}^{q}}+\left(\Psi_{3}^{2}+1\right) \frac{\partial G}{\partial \Psi_{3}}+\frac{\partial G}{\partial \psi}=0$

namely, every solution $G\left(\Psi_{1}^{q}, \Psi_{2}^{q}, \Psi_{3}, \xi_{q}, \psi\right)$ of the previous partial differential equation is an observable quantity for this system. We found the following four independent solutions:

$$
\begin{array}{rr}
A^{q} \equiv \frac{\Psi_{1}^{q}-\Psi_{3}}{1+\Psi_{1}^{q} \Psi_{3}}, & V^{q} \equiv \Psi_{2}^{q} \frac{1+\Psi_{1}^{q} \Psi_{3}}{1+\Psi_{3}^{2}}, \\
L^{q} \equiv \psi-\operatorname{atan} \Psi_{1}^{q}, & \xi_{q}
\end{array}
$$

and the local decomposition is:

$$
\left\{\begin{array}{l}
\dot{A}^{q}=\nu\left(1+A^{q^{2}}\right)\left(\xi_{q}-V^{q}\right) \\
\dot{V}^{q}=\nu A^{q} V^{q}\left(2 V^{q}-\xi_{q}\right) \\
\dot{L}^{q}=\dot{\xi}_{q}=0 \\
\beta=-\operatorname{atan} A^{q}-L^{q}+S_{p} \frac{\pi}{2}
\end{array}\right.
$$

where $S_{p}$ can be \pm 1 depending on the values of the parameters. We do not provide here this dependence. In [11] we derive some important properties relating $S_{p}$ to the robot motion.

Deriving this decomposition is very hard. As shown, it is based on the solutions of two partial differential equations. However, to check the validity of this decomposition is very simple since it only requires to compute derivatives (e.g. this can be done by using the matlab symbolic computation). Furthermore, also the solution has a simple analytical expression.

This decomposition has a very practical importance. It tells us that, when the robot accomplishes circular trajectories, the information contained in the sensor data (i.e. the information contained in the function $\nu(t)$ and $\beta(t)$ ) allows us to estimate only the state $\Theta \equiv\left[A^{q}, V^{q}, L^{q}, \xi_{q}\right]^{T}$ and not the original 
state $[\mu, \gamma, \phi, \psi, \xi, \delta, \eta]^{T}$. In the next sections we will provide a powerful strategy to estimate the initial value $\Theta_{0} \equiv\left[A_{0}^{q}, V_{0}^{q}, L^{q}, \xi_{q}\right]^{T}$ for a given circular trajectory. We remark that $\Theta_{0}$ depends on the calibration parameters and on the initial values of $\mu$ and $\gamma$. Our goal is the estimation of $\phi, \rho, \psi, \eta, \delta, \xi$, which are five parameters. By performing the estimation of $\Theta_{0}$ we obtain four independent equations on these parameters. On the other hand, we also add two new unknowns (the values of the initial $\mu$ and $\gamma$ ). Therefore, in order to have enough equations, we must combine at least three independent circular trajectories (i.e. with a different $q$ ).

\section{Analytical Properties of the Observation FUNCTION}

In this section we summarize some important properties of the observation $\beta$ obtained when the robot accomplishes circular trajectories. These properties are fundamental to introduce our calibration strategy. For the sake of conciseness, we cannot derive these properties here. However, a detailed derivation is available in [11]. Furthermore, it is possible to directly verify the validity of these properties with a simple substitution. For the sake of simplicity in the next two sections we neglect the suffix $q$ on the three parameters $A, V$ and $L$. On the other hand, to distinguish $\xi_{q}$ from $\xi$ previously defined, we still maintain $q$ in $\xi_{q}$.

First of all, it is possible to directly integrate the dynamics in (17) to get an analytical expression for $\beta$ vs the time or vs the curve length $s$ defined by the following equation:

$$
s=s(t)=\int_{0}^{t} \nu(\tau) d \tau
$$

The expression of $\beta$ vs $s$ is given by the following equations:

$$
\begin{gathered}
w=\xi_{q} \tan \left(c+S_{w} \xi_{q} s\right) \\
V=\frac{\xi_{q} k\left(2 k-\xi_{q}\right)+k w^{2}+S_{V} w \sqrt{k\left(k-\xi_{q}\right)\left(w^{2}+\xi_{q}^{2}\right)}}{\left(2 k-\xi_{q}\right)^{2}+w^{2}} \\
A=S_{y} \sqrt{\frac{k\left(2 V-\xi_{q}\right)-V^{2}}{V^{2}}} \\
\beta=\operatorname{atan}(A)-L+S_{p} \frac{\pi}{2}
\end{gathered}
$$

where $c$ and $k$ are two time-independent parameters whose value depends on the initial robot configuration with respect to the feature and $S_{w}, S_{V}$, and $S_{y}$ are three sign variables, namely, as for $S_{p}$, they can assume the value of +1 or -1 . The validity of this expression for $\beta$ can be checked by directly computing the time derivatives of $V$ and $A$ respectively in (20) and (21) and by verifying that they satisfy (17).

The variables $S_{V}, S_{y}$ and $S_{p}$ flip in correspondence of special points whose value can be determined by imposing the continuity of the expressions (19-22) and their first derivatives (see [11] for a detailed discussion of this continuity analysis).
Among these points, there are the ones where the function $w$ in (19) diverges. We call them nodes. They are:

$$
s_{n}=-S_{w} \frac{c}{\xi_{q}}+j \frac{\pi}{\xi_{q}}+S_{w} \frac{\pi}{2 \xi_{q}}
$$

$j$ being an integer. In other words, there are infinite nodes at the distance of $\frac{\pi}{\left|\xi_{q}\right|}$ one each other.

For every point $s$ we introduce an operation which associates to $s$ the point $\hat{s}$ defined by the following equation:

$$
\hat{s} \equiv 2 s_{n}^{R}-s
$$

where $s_{n}^{R}$ is the closest node to $s$ on the right. In [11] we derive the following fundamental theorem whose validity can in any case be checked by a direct substitution:

Theorem 1 (Reflection of the Observation Function) The observation function satisfies the following fundamental equation $\forall s$ :

$$
\beta(s)+\beta(\hat{s})=-2 L(\bmod \pi)
$$

This theorem is fundamental for estimating the parameters $\xi_{q}, L, k$ and $c$ related to a given trajectory (see the algorithm 1 in the next section and the videos in [23]). The name reflection comes from the fact that according with (24) $\hat{s}$ is the reflection of $s$ with respect to the node between them $\left(s_{n}^{R}\right)$.

\section{The Strategy to Estimate the System PARAMETERS}

It is possible to verify that the observation function is a periodic function whose period is:

$$
T_{S}=\frac{2 \pi}{\left|\xi_{q}\right|}
$$

Equation (26) allows us to estimate the parameter $\xi_{q}$ by evaluating the period of the observation function. Actually, this equation does not provide the sign of $\xi_{q}$. However, this sign is positive for all the values of $q<\frac{1}{\delta}$ (i.e. when the robot accomplishes counter clock-wise circular trajectories).

Once $\xi_{q}$ is estimated, the next step consists in the evaluation of the position of one node. Indeed, once we know the position of one node, we can determine $c$ (or better few candidates of $c$ ) by using (23). On the other hand, the position of one node can be evaluated by using the previous theorem (i.e. the equation (25). The algorithm 1 describes the procedure to perform this evaluation. In [23] it is possible to get some videos which illustrate how this algorithm works in simulated and real scenarios. The algorithm computes the left hand side of equation (25), called $\theta\left(s_{c}, s\right)$, for every possible node candidate $\left(s_{c}\right)$, which is in the interval $\left[0, \frac{T_{S}}{2}\right]$. The function $\theta\left(s_{c}, s\right)$ is independent of the second argument $s$ when $s_{c}=s_{n}$. Indeed, $\theta\left(s_{n}, s\right)=-2 L \forall s$. This means that the standard deviation of $\theta\left(s_{c}, s\right)$ respect to the second argument $(s)$ is zero when computed in $s_{n}$ (i.e. $\sigma\left(s_{n}\right)=0$ ). When the robot sensors are affected by measurement errors, the function $\sigma\left(s_{c}\right)$ attains its minimum on $s_{n}$ (see figure $4 e$ ). 
Algorithm 1 (Returns one Node)

$$
\begin{aligned}
& \text { for } s_{c}=0 \text { to } \frac{T_{S}}{2} \text { do } \\
& \quad \text { for } s=0 \text { to } \frac{T_{S}}{2} \text { do } \\
& \quad \hat{s}=2 s_{c}-s \\
& \quad \theta\left(s_{c}, s\right)=\beta(s)+\beta(\hat{s})(\bmod \pi) \\
& \quad \text { end for } \\
& \text { end for } \\
& \text { for } s_{c}=0 \text { to } \frac{T_{S}}{2} \text { do } \\
& \quad \sigma\left(s_{c}\right)=\text { standard deviation of } \theta\left(s_{c}, s\right) \\
& \text { end for } \\
& s_{n}=\arg \min _{s_{c}} \sigma\left(s_{c}\right)
\end{aligned}
$$

Once $s_{n}$ is determined, equations (24) and (25) allow us to immediately evaluate the parameter $L$. Furthermore, as said before, equation (23) allows us to evaluate $c$. In both cases few possible values for these parameters are actually provided. The correct ones can be selected by combining more than one circular trajectory. On the other hand, combining at least three trajectories (with different $q$ ) is necessary also to estimate our original parameters $\phi, \psi, \eta, \delta$ and $\xi$ once the parameters $\xi_{q}$ and $L$ are evaluated for each trajectory.

Once the parameters $\xi_{q}$ and $L$ are estimated for at least three independent trajectories (i.e. corresponding to three different values of $q$ ), the calibration parameters $\phi, \psi, \eta, \delta$ and $\xi$ can be found by using (11), the first equation in (14) and the third equation in (16). The method to get this estimation is simple and is explained in [11]. Finally, in [11], we also explain how to estimate the original calibration parameters (i.e. $\phi, \rho, \psi$, $\delta_{R}, \delta_{L}$ and $\delta_{B}$ ) when it is available a supplementary metric measurement consisting in the initial distance of the robot from the feature for just one among the three considered trajectories.

\section{PERformance EVAluation}

We evaluate the performance of the proposed strategy by carrying out both simulations and real experiments. In particular, since in the simulations the ground truth is available, we compare our strategy with a method based on an Extended Kalman Filter $(E K F)$. This method uses the $E K F$ to integrate the encoders data and the data provided by the camera. The matrices defining this filter can be obtained starting from the analytical expressions in (4) and (7). From now on we will refer to this calibration method with $C E K F$ (Calibration based on the $E K F$ ).

\section{A. Simulations}

We simulate a mobile robot equipped with encoder sensors and an omnidirectional camera.

Regarding the encoders we simulate their data according with the model introduced in [3]. In other words the measurements are affected by zero mean Gaussian errors independent among them. Furthermore, the variance of each measurement is proportional to the value provided by the sensor. Let us consider for instance the right wheel and let us suppose that the true rotation occurred at a given time step is equal to $\delta \alpha_{R}^{t r u e}$. We generate the following measurement: $\delta \alpha^{R}=N\left(\delta \alpha_{R}^{\text {true }}, \frac{K^{2}}{r}\left|\delta \alpha_{R}^{\text {true }}\right|\right)$, where $N\left(m, \sigma^{2}\right)$ indicates the normal distribution with mean value $m$ and variance $\sigma^{2}, r$ is the nominal value of the radius of the wheel and $K$ characterizes the non systematic odometry error. The precision of our strategy is always excellent, even when we considered values of $K$ much larger (hundred times) than the values obtained through real experiments (see [5] where $K \simeq 510^{-4} m^{\frac{1}{2}}$ ). In this section we provide a part of the results obtained with our simulations. In particular, we set $r=0.3 m, K=0.001 m^{\frac{1}{2}}$ and the distance between the wheels $B=0.5 \mathrm{~m}$.

The simulated exteroceptive sensor provides the bearings of a single feature at the origin. Furthermore, we assume that these bearing measurements are affected by a zero mean Gaussian error with a variance equal to $(0.5 \mathrm{deg})^{2}$. The small value of this variance was necessary in order to guarantee the convergence of the $C E K F$. However, in [11] we consider larger values for this variance (up to $\left.(5 \mathrm{deg})^{2}\right)$ and the performance of our strategy is always very good.

We also investigate the impact of having a path not perfectly circular. To this goal we divide the circular path in ten thousands segments. For each one we compute the corresponding displacement for the right and the left wheel $\left(\Delta s_{R}^{c}\right.$ and $\left.\Delta s_{L}^{c}\right)$. Then, we generate the displacements of the right and left wheel $\left(\Delta s_{R}\right.$ and $\left.\Delta s_{L}\right)$ randomly with a Gaussian distribution: $\Delta s_{R}=N\left(\Delta s_{R}^{c},\left(\iota \times \Delta s_{R}^{c}\right)^{2}\right)$ and $\Delta s_{L}=N\left(\Delta s_{L}^{c},\left(\imath \times \Delta s_{L}^{c}\right)^{2}\right)$. We consider three cases: Model 1 where $\imath=0$ (i.e. perfect circular trajectory), model 2 where $\imath=0.02$ and model 3 where $\imath=0.04$. In fig. 2 we plot the ratio $\frac{\Delta s_{L}}{\Delta s_{R}}$ vs time when the robot accomplishes a circular trajectory with $q=0.7$. On the left it is displayed the real case obtain by using the robot AMV-1 from the BlueBotics company (see [1] for a description of the robot). On the right it is considered the simulated robot when $\imath=0.02$. It is possible to realize that the real case satisfies the circular hypothesis better than the case when $\imath=0.02$.
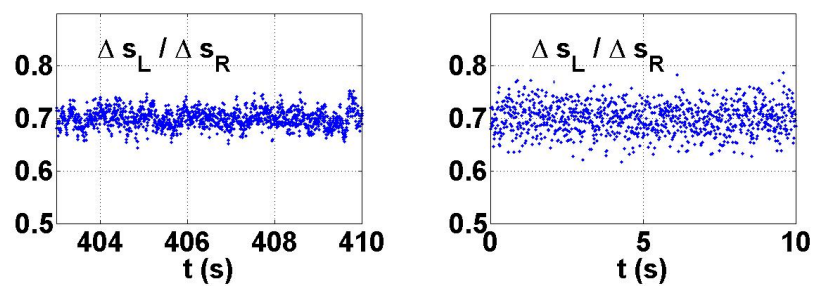

Fig. 2. The ratio $\frac{\Delta s_{L}}{\Delta s_{R}}$ vs time when the robot accomplishes a circular trajectory $(q=0.7)$. On the left the result obtained with the real robot AMV1 and on the right our simulated robot when $\imath=0.02$.

In fig. 3 we display the precision on the parameters vs the number of camera observations when the estimation is performed by the $C E K F$. In contrast with our strategy, this filter requires to initialize the parameters. We obtained that, in order to have the convergence, the initial relative error on the parameter $\eta$ must be smaller than $10 \%$. Regarding $\delta$ and $\xi$ the relative error must be smaller than $20 \%$. Finally, regarding $\phi$ and $\psi$ the initial error must be smaller than 10deg. From fig. 3 we remark that the convergence is very slow for the parameters $\phi, \psi$ and $\eta$. 

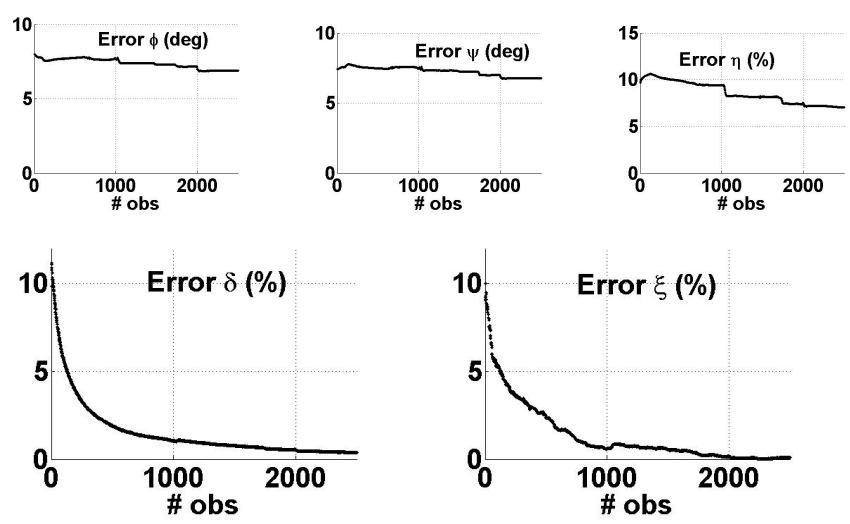

Fig. 3. The precision on the parameters estimated through $C E K F$ vs the number of exteroceptive observations

\begin{tabular}{|c|c|c|c|c|c|c|}
\hline Mod & \#Obs. & $\Delta \phi \mathrm{deg}$ & $\Delta \psi \mathrm{deg}$ & $\frac{\Delta \eta}{\eta} \%$ & $\frac{\Delta \delta}{\delta} \%$ & $\frac{\Delta \xi}{\xi} \%$ \\
\hline 1 & 250 & 0.06 & 0.05 & 0.5 & 0.02 & 0.07 \\
\hline 2 & 250 & 0.5 & 0.3 & 1.2 & 0.04 & 0.12 \\
\hline 3 & 250 & 1.1 & 0.4 & 2.7 & 0.07 & 0.14 \\
\hline$C E K F$ & 250 & 7.8 & 7.6 & 10 & 3.2 & 3.6 \\
\hline 1 & 500 & 0.06 & 0.05 & 0.4 & 0.02 & 0.05 \\
\hline 2 & 500 & 0.4 & 0.3 & 1.1 & 0.04 & 0.1 \\
\hline 3 & 500 & 0.8 & 0.3 & 1.9 & 0.06 & 0.1 \\
\hline$C E K F$ & 500 & 7.7 & 7.5 & 9.8 & 1.9 & 2.5 \\
\hline 1 & 1000 & 0.06 & 0.05 & 0.3 & 0.02 & 0.05 \\
\hline 2 & 1000 & 0.3 & 0.2 & 0.6 & 0.03 & 0.09 \\
\hline 3 & 1000 & 0.6 & 0.3 & 1.5 & 0.04 & 0.09 \\
\hline$C E K F$ & 1000 & 7.5 & 7.3 & 9.2 & 1.0 & 1.2 \\
\hline
\end{tabular}

TABLE II

THE ERRORS ON THE PARAMETERS AVERAGED ON 100 SIMULATIONS ESTIMATED BY THE $C E K F$ AND OUR STRATEGY.

Table II compares the performance of our approach with respect to $C E K F$ when the number of exteroceptive observations are 250, 500 and 1000 . Even for model 3 (i.e. when $\imath=0.04$ ) our method significantly outperforms $C E K F$.

\section{B. Real Experiments}

We used the mobile robot e-puck (see [7] for a detailed description of this robot and its sensors). In our experiments we only used the camera and the odometry sensors. Actually, our strategy has been developed to calibrate an omnidirectional bearing sensor. In contrast, the available camera, has a very limited angle of view $(\simeq 38 \mathrm{deg})$. In practice, it is in general not possible to observe a single feature during the entire circular trajectory accomplished by the robot. The only situation where this is possible occurs when the feature is inside the circular trajectory and close to the center. Furthermore, the camera must look towards the center of the circumference. This is the case when the angle $\phi$ is close to 0deg and $\psi$ is close to $90 \mathrm{deg}$ ). Since the available camera looks ahead, we fixed in front of the robot a small mirror (see figure $4 a$ ). Obviously, in these conditions our strategy cannot estimate the extrinsic calibration parameters related to the real camera. However, it estimates the parameters related to the virtual camera, i.e. the one mirrored. We remark that the goal of this

\begin{tabular}{|c|c|c|c|c|c|}
\hline Tape & $\phi(\mathrm{deg})$ & $\psi(\mathrm{deg})$ & $\eta\left(\mathrm{m}^{-1}\right)$ & $\delta$ & $\xi\left(\mathrm{m}^{-1}\right)$ \\
\hline No & -5.80 & 117.18 & 10.21 & 0.9987 & 18.99 \\
\hline Yes & -5.67 & 116.91 & 10.40 & 0.9959 & 18.97 \\
\hline
\end{tabular}

TABLE III

THE CALIBRATION PARAMETERS WITH AND WITHOUT TAPE ESTIMATED IN OUR EXPERIMENTS.

experiment is not to estimate the configuration of the true camera but to validate our strategy. Therefore, we never mind whether the camera we are considering is the virtual camera.

An issue which arises when the feature is inside the trajectory is the possibility to have collisions with the feature. In order to avoid this, the circumference has to be large. In practice we could not consider trajectories with values of $q$ smaller than 0.4 .

The robot camera provides images with resolution $60 \times 60$. Figure $4 b$ shows the robot e-puck together with the source of light we adopted as a point feature in our experiments. Figure $4 c$ is an image of the feature taken by the e-puck camera during our experiments. The images were provided at a frequency in the range $[0.5,3] \mathrm{Hz}$.

We carried out two complete experiments. In the latter we increased the radius of the right wheel by $0.062 \mathrm{~mm}$ with a piece of tape. Each experiment consists of four independent trajectories with the following values $q: 0.9,0.7,0.6,0.4$. Regarding the estimation of the parameters $\xi_{q}, L, c$ and $k$ we show in figures $4 d, 4 e$ and $4 f$ only the results for $q=0.6$ without tape. In particular, we show the observation function with the estimated nodes $(4 d)$, the function $\sigma\left(s_{c}\right)(4 e)$ and the observation functions as observed (blue points) and as estimated by our strategy (red points) $(4 f)$.
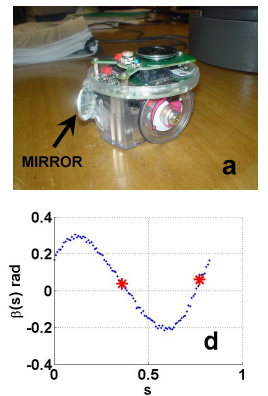
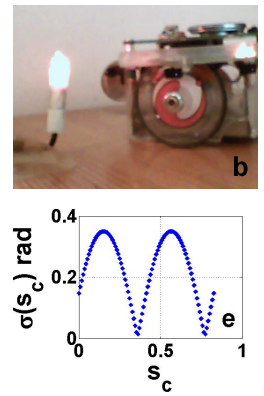
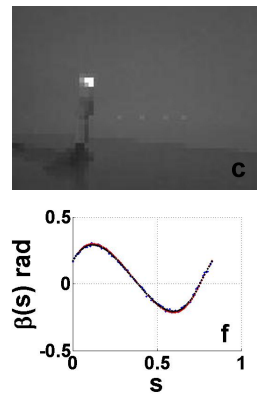

Fig. 4. The experiment performed with the robot e-puck. (a) displays the robot e-puck with a small mirror in front of the camera. The robot together with the source of light used as the feature $(b)$ and the feature observed by the robot camera $(c)$. The images below refer to an experiment with $q=0.6$ and without tape. $d$ displays the observation function with the estimated nodes, $e$ displays the function $\sigma\left(s_{c}\right)$ attaining its minima in correspondence of the nodes and $f$ displays the observation function as observed (blue points) and as estimated by our strategy (red points).

The calibration parameters with and without tape are reported in table III. Regarding the angles $\phi$ and $\psi$, we remark that the difference between the case with tape and without tape is smaller than $0.3 \mathrm{deg}$, which is roughly the mean errors obtained in our simulations. This is consistent with the fact that the tape does not affect these angle parameters. Regarding $\eta$ 
the difference is $\simeq 2 \%$ which is a bit larger than the mean error obtained in our simulations. Also regarding $\delta$ the difference is $\simeq 0.3 \%$ which is definitely larger than the mean error obtained in our simulations. This is consistent with the increased radius of the right wheel due to the tape $(0.062 \mathrm{~mm})$. In particular, since the wheel radius is $\simeq 2 \mathrm{~cm}$, we obtain $\simeq 0.06 \mathrm{~mm}$ for the radius change. The variation in the parameter $\xi$ is very small and not in the expected direction. In particular, it is $\simeq 0.1 \%$ which is roughly the mean error obtained in our simulations. This parameter should be affected by the tape since it depends on $\delta_{R}$. We believe that the tape also increased the effective distance between the wheels (i.e. the parameter $\delta_{B}$ ) making $\xi=\frac{1}{B} \frac{\delta_{R}}{\delta_{B}}$ unaffected by the tape.

\section{CONCLUSIONS}

In this paper we considered the problem of simultaneously calibrating an odometry system and the extrinsic parameters of a bearing sensor. The calibration only uses a single point feature.

Two new contributions were provided:

- A local decomposition of the considered system based on the theory of distributions developed in [9];

- A simple strategy to robustly, efficiently and accurately estimate the parameters describing both the extrinsic bearing sensor calibration and the odometry calibration.

The performance of the proposed strategy was carefully evaluated by carrying out both simulations and real experiments with the mobile robot e-puck. We found excellent results in terms of precision, robustness and facility of implementation. We remark that the proposed strategy was able to detect a variation in the wheel diameter of less than $0.1 \mathrm{~mm}$ although the robot moved along very short paths and by just observing the bearing of a single feature. We consider this an excellent result and we believe that this approach can have a huge impact on the problem of self-calibration in mobile robotics especially if it can be extended to the $3 D$ case and hence it can be applied in outdoor environment. At this regard, future works will focus on the following topics:

- extend this strategy to the $3 D$ case, i.e. remove the hypothesis of a perfect alignment of the vertical axis of the bearing sensor with the world frame vertical axis;

- extend this strategy in order to consider also range sensors and other kind of features.

Finally, we believe that the theory of distributions is a very powerful tool to face many estimation problems in the frame-work of mobile robotics and this paper is the first very successful application of this sophisticated theory.

\section{ACKNOWLEDGMENT}

This work was supported by the European Commission FP7-ICT-2007-3.2.2 Cognitive Systems, Interaction, and Robotics under the contract \#231855 (sFLY) and the EU Integrated Projects BACS - FP6-IST-027140. The author wish also to thank Jean-Marc Bollon for very useful discussions and suggestions regarding the experiment.

\section{REFERENCES}

[1] http://www.bluebotics.com/automation/AMV-1/

[2] G. Antonelli and S. Chiaverini, Linear estimation of the physical odometric parameters for differential-drive mobile robots, Autonomous Robot, July 2007, Vol 23, pages 59-68

[3] Borenstein J., Feng L., "Measurement and correction of systematic odometry errors in mobile robots," IEEE Transactions on Robotics and Automation, vol. 12, pp. 869-880, 1996.

[4] X. Brun and F. Goulette, Modeling and calibration of coupled fisheye $\mathrm{CCD}$ camera and laser range scanner for outdoor environment reconstruction, International Conference on 3D Digital Imaging and Modeling, Montreal, QC, Canada, Aug. 2123, 2007, pp. 320327.

[5] Chong K.S., Kleeman L., "Accurate Odometry and Error Modelling for a Mobile Robot," International Conference on Robotics and Automation, vol. 4, pp. 2783-2788, 1997.

[6] Doh, N. L., Choset, H. and Chung, W. K., Relative localization using path odometry information, Autonomous Robots, Vol 21, pages 143-154

[7] http://www.e-puck.org/

[8] Hermann R. and Krener A.J., 1977, Nonlinear Controllability and Observability, IEEE Transaction On Automatic Control, AC-22(5): 728-740

[9] Isidori A., Nonlinear Control Systems, 3rd ed., Springer Verlag, 1995.

[10] Martinelli A, The odometry error of a mobile robot with a synchronous drive system, IEEE Trans. on Rob. and Aut. Vol 18, NO. 3 June 2002

[11] Martinelli A, Using the Distribution Theory to Simultaneously Calibrate the Sensors of a Mobile Robot, Internal Research Report, INRIA, http://hal.inria.fr/docs/00/35/30/79/PDF/RR-6796.pdf

[12] A. Martinelli, D. Scaramuzza and R. Siegwart, Automatic SelfCalibration of a Vision System during Robot Motion, International Conference on Robotics and Automation, Orlando, Florida, April 2006.

[13] A. Martinelli and R. Siegwart, Observability Properties and Optimal Trajectories for On-line Odometry Self-Calibration, International Conference on Decision and Control, San Diego, California, December 2006.

[14] A. Martinelli, Local Decomposition and Observability Properties for Automatic Calibration in Mobile Robotics, International Conference on Robotics and Automation, Kobe, Japan, May 2009.

[15] Park F. C. and B. J. Martin, Robot Sensor Calibration: Solving AX=XB on the Euclidean Group, IEEE Trans. on Rob. and Aut., Vol 10, No 5 Oct 1994.

[16] F. M. Mirzaei and S. I. Roumeliotis, A Kalman filter-based algorithm for IMU-camera calibration: Observability analysis and performance evaluation, IEEE Trans. on Rob., 2008, Vol. 24, No. 5, October 2008

[17] Roy N., and Thrun S., Online Self-calibration for Mobile Robots, proceedings of the 1999 IEEE International Conference on Robotics and Automation, 19 May 1999 Detroit, Michigan, pp. 2292-2297.

[18] Shiu Y. C. and S. Ahmad, Calibration of Wrist-Mounted Robotic Sensors by Solving Homogeneous Transform Equations of the Form AX=XB, IEEE Trans on Rob. and Aut. Vol 5 No 1 Feb 1989

[19] R. Y. Tsai, A versatile camera calibration technique for high-accuracy $3 \mathrm{~d}$ machine vision metrology using off-the-shelf tv cameras and lenses. IEEE J. Robotics Automat, 3(4), 323-344, 1987.

[20] H.J. von der Hardt, R. Husson, D. Wolf, An Automatic Calibration Method for a Multisensor System: Application to a Mobile Robot Localization System, Interbational Conference on Robotics and Automation, Leuven, Belgium, May 1998.

[21] S. Wasielewski and O. Strauss, Calibration of a multi-sensor system laser rangefinder/camera, in Proceedings of the Intelligent Vehicles Symposium, Detroit, MI, Sept. 2526, 1995, pp. 472477.

[22] Q. Zhang and R. Pless, Extrinsic calibration of a camera and laser range finder (improves camera calibration), in Proceedings of the IEEE/RSJ International Conference on Intelligent Robots and Systems, Sendai, Japan, Sept. 280ct. 2, 2004, pp. 23012306.

[23] http://emotion.inrialpes.fr/people/martinel/Video.zip 